\title{
Criteria for Intensive Care admission and monitoring after elective craniotomy
}

Rafael BADENES ${ }^{a}$, Lara PRISCO ${ }^{b}$, Armando MARUENDA $^{a}$, Fabio Silvio TACCONE

${ }^{a}$ Department of Anesthesiology and Surgical-Trauma Intensive Care, Hospital Clinic Universitari Valencia, University of Valencia, Valencia, Spain.

${ }^{b}$ Department of Neuroanaesthesia and Neurosciences Intensive Care, John Radcliffe Hospital, Oxford University Hospitals NHS Foundation Trust, Oxford, United Kingdom.

${ }^{c}$ Department of Intensive Care, Hôpital Erasme, Université Libre de Bruxelles, Brussels, Belgium.

Correspondence to Rafael Badenes, MD, PhD, Department of Anesthesiology and SurgicalTrauma Intensive Care, Hospital Clinic Universitari Valencia, University of Valencia, Valencia, Spain.

Tel: +34 961973500; e-mail: rafaelbadenes@gmail.com

Keywords: craniotomy, ICU admission, monitoring.

Word count: 2352

\section{Key points}

Only a small proportion of elective post-operative neurosurgical patients require specific medical interventions and invasive monitoring.

Craniotomy patients have a high risk of neurological complications in the immediate postoperative period and might require advanced neuro-monitoring especially if sedation is continued in the ICU.

Postoperative clinical examination and neurological scores, bispectral index and simplified electroencephalography, and morning discharge huddles are the most used strategies in this context.

After elective craniotomy ICU admission should be warranted to patients that shows new neurological deficits, especially when these include reduced consciousness or deficits of the lower cranial nerves, or have surgical indication for delayed extubation. Currently evidence do not allow to define standardized protocol to guide ICU admission and postoperative neuromonitoring. 


\section{Purpose of review}

This article revises the recent evidence on intensive care unit (ICU) admission criteria and postoperative neuro-monitoring for patients undergoing elective craniotomy.

\section{Recent findings}

Only a small proportion of elective post-operative neurosurgical patients require specific medical interventions and invasive monitoring. Amongst these, patients undergoing elective craniotomy are frequently admitted to neuro-ICU, specialist Post-Anaesthesia Care Units (PACU) or intermediate-level care unit in the post-operative period.

Craniotomy patients have a high risk of neurological complications in the immediate postoperative period and might require advanced neuro-monitoring especially if sedation is continued in the ICU.

Furthermore, the concept of enhanced recovery after surgery (ERAS) with the goal of improving functional capacity after surgery and decreasing morbidity has expanded to encompass neurosurgery.

Postoperative clinical examination and neurological scores, bispectral index and simplified electroencephalography, and morning discharge huddles are the most used strategies in this context.

\section{Summary}

After elective craniotomy ICU admission should be warranted to patients that shows new neurological deficits, especially when these include reduced consciousness or deficits of the lower cranial nerves, or have surgical indication for delayed extubation. Currently evidence do not allow to define standardized protocol to guide ICU admission and postoperative neuromonitoring. 


\section{Introduction}

The rate of avoidable complications after neurosurgical procedures remains high [1]. A recent American database analysis including 38,058 neurosurgical patients reported an overall complication rate of $14.3 \%$, with pulmonary complications occurring in $5 \%$, cardiovascular complications in $4 \%$ and neurological complications in $3 \%$ of them [2]. Early detection and treatment of these complications often require specialist care and targeted neuro-monitoring in the early post-operative phase. Amongst these, patients undergoing Elective Craniotomy (EC) are routinely admitted to the intensive care unit (ICU), where these treatment and monitoring can be promptly provided. Nevertheless, only a small proportion of post-operative EC patients require medical interventions in the ICU.

Although common practice to stratify high risk patients relies on comorbidity score systems, a retrospective study including 400 elective patients undergoing EC identified only diabetes and older age as independent predictors for postoperative ICU admission [3].

There is large variability in the admission policy for postoperative patients in neurosurgical ICU among countries, centers, and even within the same hospital, depending on local cultural tradition but predominantly on resources. In some centers, patients are admitted for a planned 24-hour observation period following all intracranial procedures [4]. In other centers, patients are only admitted to the ICU if intra-cranial complications (i.e. early seizures, bleeding, brain oedema) develop during or immediately after surgery [5].

Moreover, as most serious complications after EC occur within the first hours after surgery, it is arguable whether post-operative destination of such patients should be revised in order to maintain optimal ICU occupancy rates [6-8] or ICU stay should be limited to the immediate post-surgical phase allowing a rapid turn-over of admissions. Among the alternative postoperative destinations of patients undergoing EC High-Density care Units (HDU), or PostAnaesthetic Care Units (PACU) provide intermediate level of care. These solutions may represent a more cost-effective allocation of ICU resources and allow the development of enhanced recovery pathways after neurosurgery $\left[9^{*}, 10\right]$.

The aim of this article is to revise recent literature exploring the criteria for planned ICU admission, perioperative complications and emergency ICU admission, existing levels of care and neurological monitoring for patients undergoing EC. 


\section{Planned ICU/PACU admission criteria}

The existing large variability on postoperative admission strategies after EC are well known at different geographical levels. A national Spanish survey aiming to describe the care pathway and post-operative treatment standards for neurosurgical interventions demonstrated that the postoperative destination of the patients is often decided by the anesthetists (73\%) with high ICU admission rates (83\%) [11*]. In many clinical contexts this frequently happens after multidisciplinary discussion with the neurosurgical team.

The joint team decision should weigh pre-operative conditions (patient comorbidities, functional level and issues related to the type and site of lesion), risk for peri-operative complications and postoperative requirements (neuro-monitoring, sedation, two-stage surgical intervention).

Careful consideration of the patients' comorbidities in the preoperative assessment is paramount, and stratification for severity is widely used in anaesthetic practice by means of the American Society of Anaesthesiologist score (ASA) [12]. The preoperative assessment should take into account chronic and stable comorbidities as well as progressive organs and systems' impairment, intracranial disease-related conditions (such as electrolytes imbalances, seizures, vasospasm, delayed cerebral ischaemia, etc.) and pharmaco-induced alterations (steroidsinduced hyperglycaemia, clotting abnormalities, arrhythmias, etc.) [12].

It is advised to plan ICU/PACU admission for patients with ASA $\geq 3$ following EC despite low level of evidence. Moreover, the presence of diabetes, advanced age (>65 y) and EC for vascular lesions are associated with higher probability of ICU/PACU admission [3].

The type and site of intracranial lesion per se represent indicators of perioperative requirements and often diverse pathologies (tumours, vascular, functional, congenital) have established different clinical pathways to follow. Intracranial vascular lesions are often associated to secondary intracranial disease (subarachnoid/intracerebral haemorrhage, vasospasm, CSF obstruction, stroke), systemic comorbidities (hypertension, smoking history, carotid stenosis, ischaemic heart disease, previous stroke and obesity), higher risk for perioperative complications (haemorrhage, haemodynamic instability, electrolytes disturbances, seizures) [13] and finally, more likely to require tight target blood pressure control in the postoperative phase [3]. On the other hand, awake EC for tumour resections and EC for grid reading in epilepsy may represent "low" risk of ICU/PACU admission and sometimes early home discharge is also considered [14].

There is no consensus on criteria for ICU admission and on the optimal timing of a postsurgical computed tomography (CT) scan in adult patients undergoing surgery for supratentorial gliomas. Of 264 patients included in a retrospective study only 21 had planned postoperative admission to the ICU (mean length of stay 19.7h) based on their clinical characteristics (Karnofsky performance status $<70$, American Society of Anesthesiologists score $(A S A)>2$ or Charlson Comorbidity Index $>5$ ) and a single case of haemorrhagic complication [15*]. 24 patients had a CT scan within 24 hours after the procedure (local oedema 
in five cases and one haematoma surgically evacuated) and two cases required unplanned ICU admission.

These data reinforce the statement that ICU admission should not be routinely planned for patients undergoing EC for supra-tentorial lesions unless pre-assessment conditions and type of lesion will benefit of enhanced recovery after surgery to facilitate uncomplicated hospital stay and prompt discharge home.

\section{Perioperative Complications and Unplanned ICU/PACU admission}

Complications after EC may be neurosurgical or systemic and their anticipation is possible awareness of the surgery details and the anaesthesia technique are essential. In a prospective series of 188 patients admitted to the ICU after EC for brain tumors resection $89 \%$ did not require postoperative sedation. Of these patients $30 \%$ developed at least one complication: $25 \%$ postoperative nausea and vomiting (PONV) and $16 \%$ neurological complications $\left[16^{* *}\right.$. The latter was highly associated with intraoperative bleeding. Furthermore, of seven patients (4\%) who required readmission to ICU after discharge $43 \%$ had posterior fossa EC.

PONV is a well recognised condition after brain surgery. Moreover, the high rate of new neurological deficit justifies the unplanned ICU admission following "moderate to high-risk" EC. Seemingly, intraoperative bleeding must be cautiously assessed as it might evolve into intracranial expanding haematomas. Finally, patients undergoing posterior fossa EC may require longer ICU admissions.

Indeed, due to the high risk of damage to cranial nerves and brainstem compression patients undergoing infratentorial craniotomy have high rates of failed extubation after surgery. In a prospective series of 2118 consecutive infratentorial EC 94 (4.4\%) failed extubation at some point during their hospital stay $\left[1^{\star}\right]$. The authors identified five independent risk factors for postoperative failed extubation: previous craniotomy, preoperative caudal cranial nerve deficit, tumor size, tumor position, and maximum change in blood pressure (BP) during the operation. On the other hand failed extubation was associated to a higher rate of pneumonia, mortality, unfavorable Glasgow Coma Scale, longer stay in ICU and hospitalization.

Although there is missing validation yet for neurosurgery, the Surgical Apgar Score (SAS) has been used as a prediction score for postoperative complications in vascular surgery. A small recent study retrospectively explored whether the application of SAS assessed by intraoperative BP, heart rate, and blood loss, in 99 patients undergoing EC for meningioma resection was accurate predictor of major postoperative complications $\left[18^{* *}\right]$. In the multivariate logistic regression model, SAS was an independent predicting factor of major complications following surgery for intracranial meningiomas (OR $0.57,95 \% \mathrm{Cl} 0.38$ to $0.87 ; p=0.009$ ), and a decrease of one mean SAS increased the rate of major complications by $43 \%$. Since this scoring system is relatively simple, objective, and practical, they suggest that SAS be included as an indicator in the guidance for the level of care after craniotomy for meningioma resection. 


\section{Levels of postoperative specialist care}

The emerging development of PACU in Europe is changing the care pathways of patients undergoing EC. Although admission to specialist neurosurgical units is associated to improved patients' outcomes after a variety of acute neurological conditions [19] the efficacy of intermediate-level of care units for some of these diseases has not been studied (except for acute stroke units) [20].

A prospective observational study recruited 200 consecutive adult patients to test the efficacy and safety of a non-ICU level of postoperative care for a selected group of patients undergoing EC $\left[9^{*}\right]$. The proposed protocol allowed to transfer patients to the neurosurgical ward after a 4-hour period of recovery in a specialist PACU following EC for supra-tentorial tumors. Criteria for early transfer included hemodynamic stability and the absence of any new post-operative neurological deficit. A small proportion of patients $(2.5 \%)$ required ICU transfer during the first 48 hours after surgery ( 3 for agitation, 1 for seizures and 1 for neurological deterioration). None of the patient immediately discharged to the neurosurgical ward experienced a major complication or prolonged hospital stay following this protocol implementation suggesting an intensive yet short-time in specialist postoperative units might be the best destination for a select proportion of patients undergoing EC.

The increasing endeavors to make inpatient treatment processes more effective leads to a reduction of the length of stay in hospital and to a minimisation of post-operative monitoring. Therefore, a National service evaluation in Germany was used to determine potential postoperative complications for neurosurgical patients undergoing elective surgery with respect to the requirement for ICU admission [21]. A total of 499 patients were scheduled for elective craniotomy over 155 neurosurgical departments. Major complication (i.e. haemorrhage, infarct, oedema, empyema and death) occurred in 19 patients (12\%), as reported in previous studies in the literature [22]. Incidence of minor complications was $38 \%$. The results of the survey showed a broad preference for ICU monitoring of patients undergoing elective craniotomy in Germany. Authors conclude that the gold standard of postoperative monitoring of neurosurgical patients undergoing elective surgery is still the ICU because a rapid and specific intervention was required in more than $20 \%$ of patients. Although more flexible surveillance modalities are available, a cost-driven restructuring of postoperative monitoring and in particular a reduction of the stay in hospital must be subjected to detailed evaluation.

Whether the local strategy is ICU or PACU admission, level and specialist training of staff is essential in the management of the postoperative neurosurgical patient. A recent observational study aimed to evaluate the impact of a specialist in neurocritical care medicine (i.e. a physician with neurocritical care experience as well as training in neurology, anesthesiology, or general intensive care medicine) who could help for clinical management of patients with a variety of neurological and neurosurgical conditions, on safety and outcome in a neuroscience intermediate-level care unit during weekday daytime hours [23*]. This before-after study of PACU implementation with specialist medical staff was performed over three years (1 year before implementation, 2 years after). Of the 2931 patients eligible 586 were studied (post- 
operative surveillance for brain tumors). Following the implementation, there was a significant reduction in discharge time from PACU to the ward, as well as the reduction in the mean length of stay (LOS). No significant safety concerns were identified. This study demonstrated that the optimisation of a neuroscience intermediate-level care unit involving co-management of patients by a neurocritical specialist can improve safety of patients' flow. These findings are of particular interest as pressure on economic resources use requires a careful rationalisation of services.

\section{Postoperative Monitoring}

Neurosurgical patients are at risk of neurological complications in the immediate postoperative period [24] and require specialized neurological monitoring (Table I). A prospective observational study examined the impact of the Ramsay scale, Canadian Neurological Scale (CNS), Nursing Delirium Screening Scale (Nu-DESC) and Bispectral Index (BIS) in comparison with the assessment of pupils and Glasgow Coma Scale (GCS) to detect early post-operative neurological complications in PACU [25*]. Among 70 patients, 16 patients (23\%) developed neurological complications. The scales-BIS were more sensitive $(94 \%$ vs. $50 \%)$ and allowed a more precise estimation of neurological complications in PACU than clinical examination ( $p=$ $0.002 ; \mathrm{OR}=7.15,95 \% \mathrm{Cl}=2.1-24.7$ vs. $\mathrm{p}=0.002 ; \mathrm{OR}=9.5,95 \% \mathrm{Cl}=2.3-39.4$ ). These results suggested that combined assessment of pupils, GCS, Ramsay scale, CNS, Nu-DESC and BIS improved early detection of postoperative neurological complications in PACU after elective craniotomies.

Post-operative clinical neuro-monitoring varied according to the type of surgery, although strength and sensitivity were explored in between $70-80 \%$. The authors' conclusion highlighted a great variability in the attitude for neurological monitoring in these patients, due to the absence of guidelines, to the different resources in terms of staff and equipment, and the type of surgery $\left[11^{*}\right]$.

Morning discharge huddles consist of a multidisciplinary decision from the members of the medical team and are used to improve communication and patient care and to facilitate patient flow through the hospital. A study investigated how a neurosurgeon leading interdisciplinary daily morning huddle affected various costs of patient care and patient satisfaction [26]. Huddles were conducted at 8:30 am Monday through Friday, and lasted approximately 30 minutes; results were compared in the period before and after implementation of such strategy. There was a significant decrease in the number of ICU days, mean laboratory and pharmacy costs per patient after the huddle was implemented. This study showed that interdisciplinary huddles can result in potentially substantial reductions in costs per patient as well as improved patient satisfaction.

Moreover, clinical observation was sufficient to predict early post-operative complications. A CT scan before 24 hours after surgery is not recommended in the absence of clinical worsening $\left[15^{*}\right]$. 


\section{Conclusions}

The criteria for post-operative admission to ICU, high-dependency unit or a specialized neurosurgical ward vary from institution to institution depending on local structures and characteristics of the available resources $[27,28]$. We propose a summary of criteria for ICU admission (Table II). However, due to the lack of high quality evidence on this topic $[29,30]$, these criteria represent experts' suggestions and patient's care in this context has to be assessed on a case-to-case basis. Needless to highlight, we need better evidence to standardize the treatment and the degree of monitoring needed during the postoperative period after EC. It is highly recommended to implement clinical vigilance in the immediate postoperative period in these patients regardless of their discharge destination (ICU, PACU or ward). A brain CT scan should be obtained rapidly in the case of neurological deterioration to exclude intracranial complications. Communication between neurosurgeons, neuroanaesthestists and neurointensivists remains a critical step to optimise perioperative management of neurosurgical patients.

\section{Acknowledgements}

None.

Financial support and sponsorship

None.

\section{Conflicts of interest}

There are no conflicts of interest.

\section{BIBLIOGRAPHY}

1. Badenes R, Gruenbaum SE, Bilotta F. Cerebral protection during neurosurgery and stroke. Curr Opin Anaesthesiol 2015;28:532-6.

2. Rolston JD, Han SJ, Lau CY, Berger MS, Parsa AT. Frequency and predictors of complications in neurological surgery: national trends from 2006 to 2011. J Neurosurg 2014;120:736-45.

3. Hanak BW, Walcott BP, Nahed BV, et al. Postoperative intensive care unit requirements after elective craniotomy. World Neurosurg 2014;81:165-172.

4. Beauregard CL, Friedman WA: Routine use of postoperative ICU care for elective craniotomy: a cost-benefit analysis. Surg Neurol 2003;60:483-489.

5. Zhou JC, Pan KH, Huang X, et al. Delayed admission to ICU does not increase the mortality of patients post neurosurgery. Int J Neurosci 2015;125:402-408.

6. Awad IA. Intensive care after elective craniotomy: 'all politics is local'. World Neurosurg 2014; 81:64-65.

7. Hecht N, Spies C, Vajkoczy P. Routine intensive care unit-level care after elective craniotomy: time to rethink. World Neurosurg 2014;81:66-68. 
8. Tierney LT, Conroy KM. Optimal occupancy in the ICU: a literature review. Aust Crit Care 2014;27:77-84.

9*. Florman JE, Cushing D, Keller LA, Rughani Al. A protocol for postoperative admission of elective craniotomy patients to a non-ICU or step-down setting. J Neurosurg 2017;3:1-6.

A prospective observational study tested the efficacy and safety of a non-ICU level of postoperative care for a selected group of patients undergoing EC. It concluded that Care of patients undergoing uneventful elective supratentorial craniotomy for tumor on a neurosurgical floor after 4 hours of PACU monitoring appears to be a safe practice in this patient population.

10. Hagan KB, Bhavsar S, Raza SM, et al. Enhanced recovery after surgery for oncological craniotomies. J Clin Neurosci 2016;24:10-6.

11*. Valero R, Carrero E, Fàbregas N, Iturri F, Saiz-Sapena N, Valencia L; Sección de Neurociencia de la Sociedad Española de Anestesiología y Reanimación. National survey on postoperative care and treatment circuits in neurosurgery. National survey on postoperative care and treatment circuits in neurosurgery. Rev Esp Anestesiol Reanim. 2017 Mar 16. pii: S0034-9356(17)30027-0. [Epub ahead of print].

A National survey of postoperative care in neurosurgery showed that There is great variability in the responses, probably attributable to the absence of guidelines, different structures and hospital equipment, type of surgery and qualified personnel.

12. Reponen E, Korja M, Niemi T, Silvasti-Lundell M, Hernesniemi J, Tuominen H. Preoperative identification of neurosurgery patients with a high risk of in-hospital complications: a prospective cohort of 418 consecutive elective craniotomy patients. J Neurosurg 2015;123:594-604.

13. Dasenbrock HH, Smith TR, Rudy RF, Gormley WB, Aziz-Sultan MA, Du R. Reoperation and readmission after clipping of an unruptured intracranial aneurysm: a National Surgical Quality Improvement Program analysis. J Neurosurg 2017;7:1-12.

14. Venkatraghavan L, Bharadwaj S, Au K, Bernstein M, Manninen P. Same-day discharge after craniotomy for supratentorial tumour surgery: a retrospective observational single-centre study. Can J Anaesth 2016;63:1245-57.

15*. Altieri R, Cofano F, Agnoletti A et al. Postoperative Care of Patients with High-grade Glioma: Is There a Real Need for the Neurocritical ICU and Early CT Scan? J Neurol Surg A Cent Eur Neurosurg. 2017 Mar 24. [Epub ahead of print]

In this retrospective nonrandomized study demonstrated that that ICU should not always be used after craniotomy for supratentorial gliomas. Clinical observation was sufficient to predict early postoperative complications. A CT scan before 24 hours after surgery is not recommended in the absence of clinical worsening.

$16^{\star \star}$. Lonjaret L, Guyonnet M, Berard E et al. Postoperative complications after craniotomy for brain tumor surgery. Anaesth Crit Care Pain Med. 2016 Oct 4. pii: S2352-5568(16)30164-3. [Epub ahead of print]

This prospective, observational and analytic study observed that postoperative complications, especially PONV, are frequent after brain tumour surgery. Moreover, $16 \%$ of patients presented a neurological complication, probably justifying the ICU postoperative stay for early detection. 
The absence of preoperative motor deficit and intraoperative bleeding seems to predict postoperative neurologic complications. Finally, patients may present complications after ICU discharge, especially patients with fossa posterior surgery, suggesting that ICU hospitalization may be longer in this type of surgery.

$17^{*}$. Cai YH, Wang HT, Zhou JX. Perioperative Predictors of Extubation Failure and the Effect on Clinical Outcome After Infratentorial Craniotomy. Med Sci Monit 2016;22:2431-8.

In this prospective observational study, History of craniotomy, preoperative lower cranial nerve dysfunction, tumor size, tumor position, and maximum change in BP during the operation were independent risk factors related to postoperative failed extubation in patients submitted to infratentorial craniotomy.

$18^{* *}$. Hsu SY, Ou CY, Ho YN, Huang YH. Application of Surgical Apgar Score in intracranial meningioma surgery. PLoS One 2017;12:e0174328.

A retrospective study showed that SAS is an independent predictor of major complications in patients undergoing intracranial meningioma surgery, and provides acceptable risk discrimination. Since this scoring system is relatively simple, objective, and practical, they suggest that SAS be included as an indicator in the guidance for the level of care after craniotomy for meningioma resection.

19. Fuller $G$, Pallot $D$, Coats $T$, Lecky $F$. The effectiveness of specialist neuroscience care in severe traumatic brain injury: a systematic review. Br J Neurosurg 2014;28:452-60.

20. Rodgers $\mathrm{H}$, Price $\mathrm{C}$. Stroke unit care, inpatient rehabilitation and early supported discharge. Clin Med (Lond) 2017;17:173-177.

21. Henker C, Schmelter C, Piek J. Complications and monitoring standards after elective craniotomy in Germany. Anaesthesist. 2017 Mar 13. [Epub ahead of print].

22. Reponen E, Tuominen H, Hernesniemi J, Korja M. Modified Rankin Scale and Short-Term Outcome in Cranial Neurosurgery: A Prospective and Unselected Cohort Study. World Neurosurg 2016;91:567-573.

23*. Quimby AE, Shamy MC, Rothwell DM, Liu EY, Dowlatshahi D, Stotts G. A Novel Neuroscience Intermediate-Level Care Unit Model: Retrospective Analysis of Impact on Patient Flow and Safety. Neurohospitalist 2017;7:83-90.

This retrospective study has demonstrated that the optimization of a neuroscience intermediatelevel care unit involving comanagement of patients by a neurocritical specialist can reduce wait times to admission and lengths of stay, with preserved safety outcomes.

24. Badenes R, García-Pérez ML, Bilotta F. Intraoperative monitoring of cerebral oximetry and depth of anaesthesia during neuroanesthesia procedures. Curr Opin Anaesthesiol 2016;29:57681.

25*. Herrero S, Carrero E, Valero R, Rios J, Fábregas N. Postoperative surveillance in neurosurgical patients - usefulness of neurological assessment scores and bispectral index. Braz J Anesthesiol 2017;67:153-165. 
In this prospective observational study showed that applied together, the assessment of pupils, GCS, Ramsay scale, CNS, Nu-DESC and BIS improved early detection of postoperative neurological complications in PACU after elective craniotomies.

26. Chan AY, Vadera S. Implementation of interdisciplinary neurosurgery morning huddle: costeffectiveness and increased patient satisfaction. J Neurosurg 2017;7:1-4.

27. Zhou JC, Pan KH, Huang X, Yu WQ, Zhao HC. Delayed admission to ICU does not increase the mortality of patients post neurosurgery. Int J Neurosci. 2015;125:402-8.

28. Siegemund $M$, Steiner LA. Postoperative care of the neurosurgical patient. Curr Opin Anaesthesiol 2015;28:487-93.

29. Bui JQ, Mendis RL, van Gelder JM, Sheridan MM, Wright KM, Jaeger M. Is postoperative intensive care unit admission a prerequisite for elective craniotomy? J Neurosurg 2011;115:1236-41.

30. Rhondali $\mathrm{O}$, Genty $\mathrm{C}$, Halle $\mathrm{C}$, et al. Do patients still require admission to an intensive care unit after elective craniotomy for brain surgery? J Neurosurg Anesthesiol 2011;23:118-23. 